Weyrich, Steven; Sprenger, Sina; Böttrich, Marcel; Schmidt, Philipp; Laqua, Daniel; Ley, Sebastian ; Husar, Peter:

\title{
Development of a phantom to modulate the maternal and fetal pulse curve for pulse oximetry measurements
}

Zuerst erschienen in: Biomedical Engineering = Biomedizinische Technik. - Berlin [u.a.] : de Gruyter. - 57 (2012), Suppl. 1, Track-O, p. 803-806.

Erstveröffentlichung: $\quad$ 2012-09-06

ISSN (online): $\quad$ 1862-278X

ISSN (print): $\quad$ 0013-5585

DOI: $\quad$ 10.1515/bmt-2012-4204

[Zuletzt gesehen: 2019-08-15]

„Im Rahmen der hochschulweiten Open-Access-Strategie für die Zweitveröffentlichung identifiziert durch die Universitätsbibliothek IImenau."

"Within the academic Open Access Strategy identified for deposition by Ilmenau University Library."

„Dieser Beitrag ist mit Zustimmung des Rechteinhabers aufgrund einer (DFGgeförderten) Allianz- bzw. Nationallizenz frei zugänglich."

"This publication is with permission of the rights owner freely accessible due to an Alliance licence and a national licence (funded by the DFG, German

Research Foundation) respectively." 


\title{
Development of a Phantom to Modulate the Maternal and Fetal Pulse Curve for Pulse Oximetry Measurements
}

\author{
S. Weyrich ${ }^{1,2}$, S. Sprenger ${ }^{1}$, M. Böttrich ${ }^{1}$, P. Schmidt ${ }^{1}$, D. Laqua ${ }^{1}$, S. Ley ${ }^{1}$, P. Husar ${ }^{1}$, \\ ${ }^{1}$ Department of Biosignal Processing, Ilmenau University of Technology, Ilmenau, Germany, \\ 2E-Mail: steven.weyrich@tu-ilmenau.de
}

\begin{abstract}
For getting reliable information about the state of health from the fetus and the mother during labor and delivery, a fetal pulse oximeter is being developed. This paper describes the development of a special phantom to verify the algorithms for separating the weak fetal from the dominant maternal optical signal. To reach a realistic behavior it is necessary for two circulations to be controlled independently. Inspired by the natural blood circulation behavior, a RC-System with fixed resistance and capacity was created.

Pumping a liquid with a roller pump into the tubes widens them. The digital signal processor (DSP) controls the expansion of the tube diameter by modifying the rotation velocity of the pump for active regulation. Therefore a soft and good enlargeable material was used. The measured values of the pressure sensor enabled active feedback for motor control

The determined system characteristics are used to adapt the given pulse curve to the real system behavior. Via a LabView interface it is possible to change curve parameters like amplitude and frequency.

The goal was to replicate the pulsation of the blood vessels like in the abdomen of a pregnant woman. Changing the parameters influences the modulation of the signal under consideration of the transfer function. With this phantom it is possible to simulate different scenarios, for example different states of pregnancy or pathogen indications.
\end{abstract}

\section{Introduction}

Through the increased use of pulse oximeters many lives could be saved in the last few decades. The measurement of the blood oxygen level at the finger is nowadays a clinical standard. The next step is to enlarge the functional principle to unborn babies.

With such a system it would be possible to check the fetus oxygen level during pregnancy and birth. So it is easy to get a good overview about the fetal health status. Today in delivery rooms only a CTG System is used to monitor the fetal heart rate. But birth stress makes this value unreliable [1]. At this point the oxygen content of the blood could be used to notice an undersupply with oxygen in the fetus. When such a case occurs, the medical team can react in time and avoid adverse effects.

Therefore the sensor for the fetal pulse oximeter is described in [2]. For testing and verifying the separation algorithm to split the strong maternal signal from the weak fetal, a physical-like phantom is needed. To achieve a physiological behavior, two independent circuits for simulating the behavior and the interactions between the signals from the maternal and fetal blood vessels must be accurately detected.

The oxygen content of the blood is measured by the different attenuation of the radiated red and infrared light depending on the pulse rate. Therefore it is important that the relative extension of the tube corresponds to the natural behavior of the fetal blood vessels.

In [3] and [4] the extension of the fetal aorta was determined. The highest value is reached between the 33 th and 36th week of pregnancy with $14.8 \%$.

In [5], [6] and [7] different kinds of phantoms to simulate the pulsation of blood vessels for testing pulse oximeters are described. All of them only use one blood circuit, thus separation algorithms could not be tested with these systems. Therefore it is necessary to construct a phantom with two separate blood circuits and a physiological-like tube extension.

\section{Methods}

\subsection{System concept}

For modeling the transient pulse wave behavior in the human body, a model with the same temporal behavior is needed. The model used for the pulse oximeter phantom is shown in Figure 1.

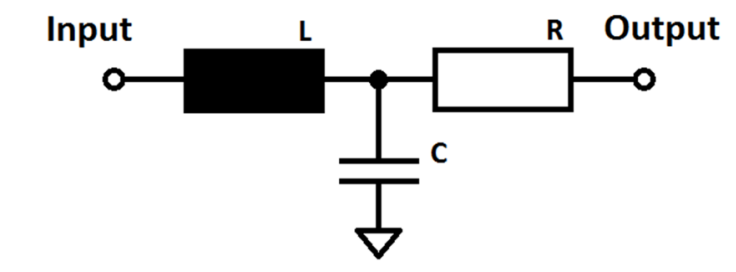

Figure 1: The model of an artery consists of an inductance L, a capacitance $\mathrm{C}$ and a resistance $\mathrm{R}$.

Referring to the model, energy is stored in the inductance $\mathrm{L}$ and the capacitance $\mathrm{C}$. After the energy supply stops, stored energy is discharged over the resistance R. A phantom with individually controllable circuits to simulate the blood vessels was developed. The application of the model is shown in Figure 2. A roller pump simulates the heart and represents the energy source. This type of pump has the advantage that the rolls prevent a reflow back to the reservoir. To be able to use different pulse rates, each circuit has its own roller pump and driver unit. 


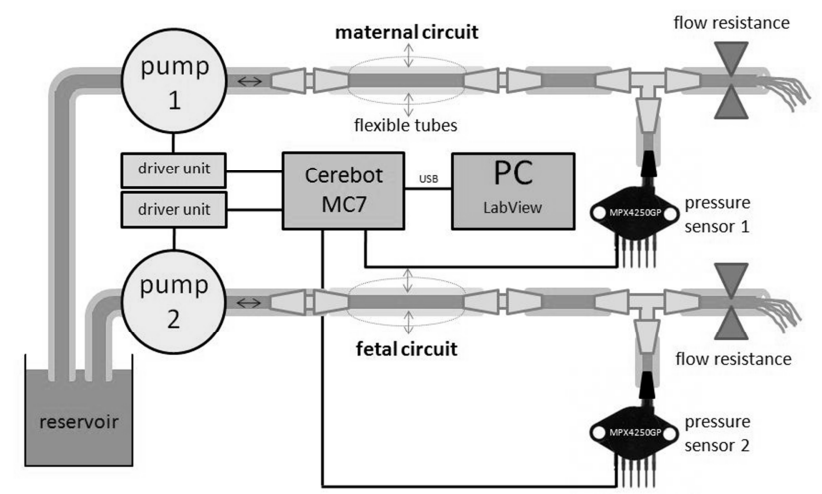

Figure 2: The central controlling unit is the Cerebot MC7 Board controlled by a PC. There are two independent driver units for driving the pumps used. Each of the two tube systems consists of a flexible tube, a flow resistance and a pressure sensor. The signal of the pressure sensors are used for active regulation of the hose diameter.

The pressure in each circuit is monitored by a pressure sensor. When the preset maximum pressure is reached, the pump is stopped. The capacitive stored energy in the tube diameter extension is now released through the flow resistance at the other end of the circuit. This resistor directly influences the time constant of the system and must be applied to reach a real pulse curve.

For radiographic measurements with the fetal pulse oximeter, only a small part of the tube is transilluminated. To maximize the output signal, two types of tubes are used. The one is highly flexible and can be enlarged easily by small pressures. The other is very rigid and hardly enlargeable.

Based on the knowledge of the relationship between the extension of the tube and the pressure in the tube, the pressure sensor is sufficient for monitoring the extension of the tube.

The pressure sensors are connected to the digital signal processor on the Cerebot MC7 board, which controls the roller pumps. The structure of the system forms the basis for the regulation process. Via a LabView program the parameters of the pulse wave, like dilation and pulse rate, can be changed.

\subsection{Instrumentation}

\subsubsection{Tube}

To get a natural behavior, the tube used for the pulse oximeter measurements must be evenly enlargeable up to $15 \%$ of its diameter within the time for half of one pulse. With this requirement different tubes are investigated. Two of them consist of silicon with a wall thickness of 0.5 and $1 \mathrm{~mm}$, respectively. The third tube is made of latex and has a wall thickness of $1 \mathrm{~mm}$.

At pressures above $110 \mathrm{kPa}$ the thinner silicon and the latex tube forms a balloon, where most of the liquid accumulates in. This behavior makes it impossible to perform reproducible measurements. The thicker tube does not show this behavior even up to pressures over $200 \mathrm{kPa}$. For that reason only the silicon tube with $1 \mathrm{~mm}$ thickness was useable to generate a constant and reproducible extension.
For the previously described rigid tube the recommended roller pump tube is used. Its wall thickness with 4 $\mathrm{mm}$ and high mechanical stability also avoid large extensions at high pressures.

\subsubsection{Pump / Motor}

To create the pulsating waveform of the arteries, we used a roller pump from Watson- Marlow, driven by a stepper motor. The step motor driver from Nanotec was used to control the motor with high precision.

\subsubsection{Motor control}

To model a natural like systole and diastole, the roller pump powering stepper motor must be controlled with a high timing precision. This task is done by a digital signal controller dsPIC33F from Microchip as the central controlling unit. It is placed on the CerebotMC7 shown in the middle of Figure 2. The DSP's main task is to modulate the speed of the connected stepper motors by using an adjustable pulse frequency modulation; thus the flow of the fluid follows the physiological example. The DSP used a basic time line to start the pulse curves on every subsystem with a constant interval. Therefore the digitalized signal of a pressure sensor is used in every circuit for implementing a pressure based control to turn off the stepper motor when the preset voltage is reached. This approach is based on the results of our experiments.

The rising slope of the arterial pressure wave was created by running the motor at the maximum reachable speed. When the pressure exceeds the preset maximum value, the motor was first decelerated and then stopped. While the acceleration was fixed at maximum, the deceleration could be adapted for varying the duration and the slope of the curve at maximum range. After the pressure dropped below the preset minimum value, the motor started running again with adjusted speed until the next pulse was generated.

\subsubsection{Sensor}

Two pressure sensors from Freescale were used for monitoring the system. The sensor allows a pressure measurement up to $250 \mathrm{kPa}$. The output range reaches from $0.2 \mathrm{~V}$ to $4.9 \mathrm{~V}$, and the resulting sensitivity is $18.8 \mathrm{mV} / \mathrm{kPa}$. The sensor is a gauge sensor, which means that the pressure is measured against atmospheric pressure.

At low pressure values water is nearly incompressible so the pressure sensor can be placed anywhere in the circuit. To reduce the influence of the pump the sensor is placed behind the silicon tube via a T-piece.

\subsection{Experimental setup}

\subsubsection{Ultrasonic measurement}

To measure the stress- expansion curve, a medical ultrasonic device was used. In Figure 3 the experimental setup is shown. 


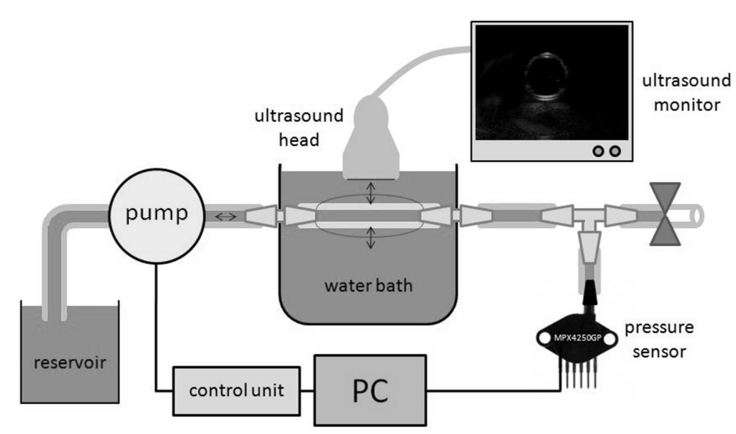

Figure 3: The ultrasonic experimental setup consists of the tubing system with a pressure sensor and a pump. The system is controlled by a control unit and PC. The ultrasound head is placed over the flexible tube lying in a water bath. These measurements are performed while a valve at the end of the tubing system is closed.

Water is a good sonic conductor, so the tube is placed into a small water pool. The ultrasonic head is placed over the tube to measure the axial extension change. In $13 \mathrm{kPa}$ steps started by $2.7 \mathrm{kPa}$ pressure the extension was measured via the ultrasonic device. Its accuracy is about \pm 0.05 $\mathrm{mm}$ tube extension. An additional measuring error results from the subjective set inner tube boarders. This is reduced by a series of measurements. The ultrasonic speeds differ in water $\left(1480 \mathrm{~m} / \mathrm{s}\right.$ at $\left.20^{\circ} \mathrm{C},[8]\right)$ from the calibrated one in the device $(1540 \mathrm{~m} / \mathrm{s},[8])$. This is why a correction factor of 0.961 has to be applied. When considering all of these facts, the accuracy of the tube extension is about \pm $0.1 \mathrm{~mm}$. The measurement uncertainty for the relaxed inner diameter of $5 \mathrm{~mm}$ is about $2 \%$ and decreases for higher extensions.

\subsubsection{Photoplethysmography}

To validate the behavior of the system during photoplethysmographic measurements, the experimental setup inFigure 4 was used.

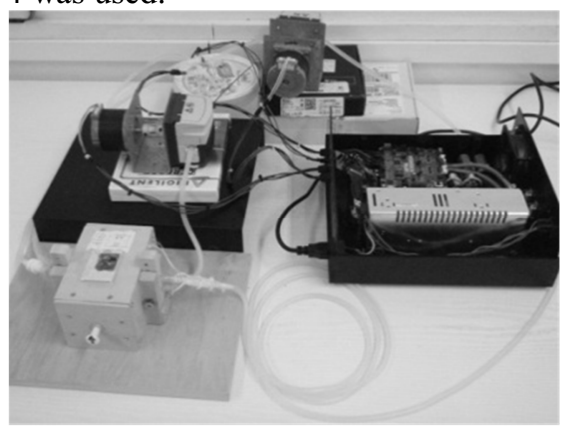

Figure 4: The experimental setup used for photoplethysmographic measurements consists of the tubing system placed in a wooden box and an infrared LED and phototransistor placed above them.

An infrared LED and a phototransistor are placed above the tubes, for measuring the reflected light. A wooden box is used to define the distances and to minimize the influence of the ambient light.

The motor control software was used for each measurement with different pule rates and extensions for each subsystem. Instead of the physiological-like liquids in [2], water was used. According to the physiological conditions, the second tube is placed above the outer with a space of $2 \mathrm{~cm}$. Furthermore, the two systems are driven with different heart rates.

\section{Results}

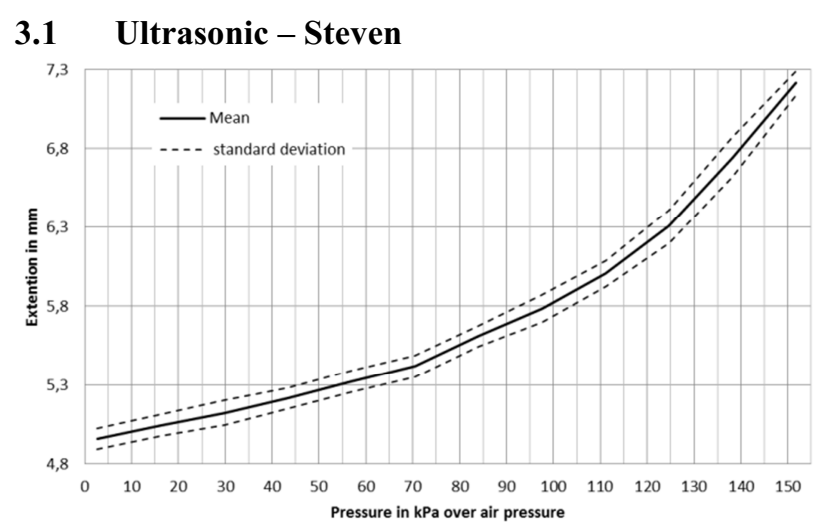

Figure 5: The graph of the Relationship between pressure and tube diameter is shown.

Figure 5 shows the connection between extension and pressure of the silicon tube. Here the mean and the standard deviation of a measurement series are printed. In the area between 5 and $5.4 \mathrm{~mm}$ the extension grows nearly linear with the pressure. To calculate pressures for extensions above an exponential curve, the fit is used.

There is a linear correlation between the extension and the pressure in the area up to $70 \mathrm{kPa}$. To calculate pressures for extensions above an exponential curve, the fit is used. The experiments yielded an accuracy of $2.7 \%$ for controlling the extension of the tube by the pressure.

\subsection{Photoplethysmography}

In Figure 6 the result of the photoplethysmographic measurement of one silicon tube is shown.

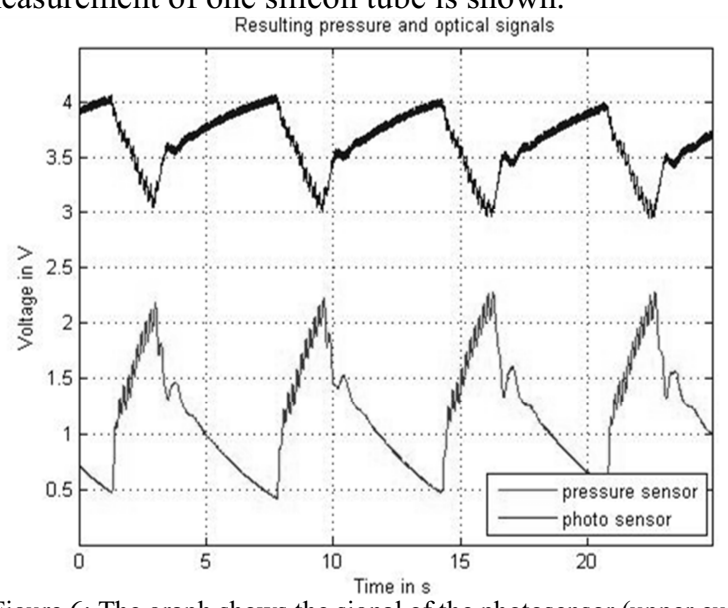

Figure 6: The graph shows the signal of the photosensor (upper curve) for one silicon tube amplified by 10 and the signal of the pressure sensor (lower curve) of the tubing system.

In the upper curve the received infrared light on the photo sensor is shown. With higher tube extension, its surface lit with infrared light also enlarges. The absorption of light therefore increases, while the amount of reflected and measured light decreases. The upper curve shows the pressure sensor signal. Its form is a good approximation to the pulse curve 


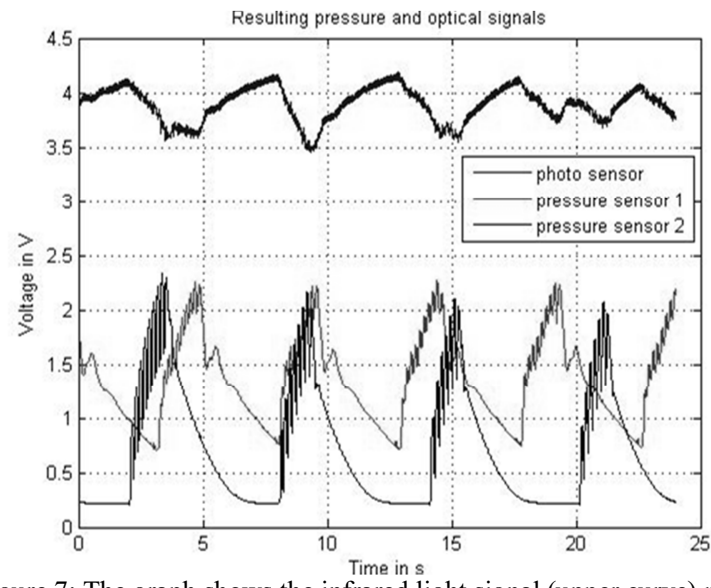

Figure 7: The graph shows the infrared light signal (upper curve) modulated by two tubing systems and amplified by 10 and the pressure signals (two lower curves) of each tubing systems.

The phototransistor signal with two circuits could be found in

Figure 7. Like in Figure 6, the received infrared radiation (upper curve) decreases at every pulse peak. When pulses of both maternal (lower curve) and fetal (middle curve) circuit occur simultaneously, the infrared curve decreases to a local minimum.

The main problems are the ripple of the pumps when the tubes are enlarged and the long rise time in general. These two factors are results of the roller pump characteristics and the missing stepper motor torque at high speed for overcoming the resistance of the pump.

The ripple causes variations of the extension despite the pressure based motor control. The maximum pressure could be reached with an accuracy of $5 \mathrm{kPa}$.The maximal pulse rate is limited by the reachable speed of the pump at higher extensions.

The system allows a maximum pulse rate of $60 \mathrm{bpm}$ at maximum extension of $25 \%$ and $90 \mathrm{bpm}$ at physiological extension of $15 \%$.

\section{Conclusion and future work}

With the developed fetal pulse oximeter, phantom physical like pulse curves can be generated. The extensions and pulse rates of the maternal and fetal circuits are individually adjustable by a LabView interface. A highly enlargeable silicon tube with $1 \mathrm{~mm}$ wall thickness was characterized to get its stress- extension -curve with an exponential growth.

To control the tube extension, a pressure based stepper motor control was implemented. The individual control for every subsystem is able to generate stable pulse durations for different pulse rates. The tube preset extension is recalculated in a maximum pressure. The implemented control stops the pump when reaching the inner tube pressure. But the maximum pressure differs in every pulse because of the strong pump influence. Also, the maximum reachable pulse rates are lower than its natural equivalent. Here the stepper motor's torque is too low at higher rounds per minute.

The next step in the phantom development process is to embed the tubes in physiological like material and to use the blood substitute described in [2]. To reach a higher accuracy, a pump and a motor with higher torque have to be used.

\section{References}

[1] C. East, B. S.P., J. King, F. C. Chan und P. Colditz, „The effect of intrapartum fetal pulse oximetry, in the presence of a nonreassuring fetal heart rate pattern, on operative delivery rates: A multicenter randomized,controlled trial,“ American Journal of Obstetrics \& Gynecology, Bd. 194, pp. 606-16, 2006.

[2] S. Ley, D.Laqua und P. Husar, „Conception of a sensor circuit for non-invasive fetal pulse oximetry based on investigations of the optical transmission properties of biological tissues," in World Congress on Medical Physics and Biomedical Engineering, Beijing, China, 2012.

[3] H. M. TONGE, Blood Flow Measurements in the Fetal Descending Aorta: Technique and Clinics.

[4] H. Tonge, Vascular dynamics in the descending aorta of the human fetus in normal late pregnancy.

[5] S. K. Nadkarni, G. Mills, D. R. Boughner und A. Fenster, „A Pulsating Coronary Vessel Phantom for Two- and ThreeDimensional Intravascular Ultrasound Studies, "Canada, 2001.

[6] M. N. H. S. Norbert Stuban, „Phantom with Pulsatile Arteries to Investigate the Influence of Blood Vessel Depth on Pulse Oximeter Signal Strength,“2012.

[7] K. Y. Oura, „Calibration System for Pulse Spectrophotometry,“ Minneapolis.

[8] C. Zink, Ultraschall-Lexikon, Berlin: Blackwell Wiss.-Verl., 1996. 